\title{
Identifying Obstructive Sleep Apnea Subtypes and Its Correlation With Mood States
}

\author{
Hyo Jin Kim', Jae-Won Choi, ${ }^{1,2}$, Eun-Jeong Joo 2,3, \\ Kyu Young Lee ${ }^{1,2}$, Soo-Young Bhang ${ }^{1,2}$, and Eui-Joong Kim ${ }^{1,2}$ \\ 'Department of Psychiatry, Nowon Eulji Medical Center, Eulji University, Seoul, Korea \\ ${ }^{2}$ Department of Neuropsychiatry, Eulji University School of Medicine, Daejeon, Korea \\ ${ }^{3}$ Department of Psychiatry, Uijeongbu Eulji Medical Center, Eulji University, Uijeongbu, Korea
}

\begin{abstract}
Objective: It has been difficult to establish specific subtypes of obstructive sleep apnea (OSA) due to the heterogeneous nature of the disorder. Previous studies have attempted to exact clinical subgroups or phenotypes of the disorder. However, the psychiatric impact of OSA has been often overlooked, and thus, this study used cluster analysis to examine subgroups of OSA incorporating mood states. Methods: This study is comprised of 346 adult OSA patients (apnea-hypopnea index, AHI $\geq 5$ ) who underwent polysomnography at Nowon Eulji Medical Center from January 2003 to November 2012. Their data also included validated self-report questionnaires that evaluate daytime sleepiness, sleep quality, morningness-eveningness, and mood states: Epworth Sleepiness Scale, Korean version of the Pittsburgh Sleep Quality Index (PSQI-K), Horne and Östberg Questionnaire, and Korean edition of Profile of Mood States (K-POMS), respectively. A K-means cluster analysis was performed to determine the optimal number of clusters and characteristics. Results: We identified three clusters: cluster 1 , moderately symptomatic OSA with the least disturbed mood ( $n=166)$; cluster 2, severely symptomatic OSA not so disturbed mood ( $n=90)$; cluster 3 , moderately symptomatic with highest disturbed mood $(n=90)$. Cluster 1 had the lowest AHI and the lowest K-POMS total scores. Cluster 2 , despite having the most severe OSA parameters, did not show mood disturbances. Cluster 3 had the highest K-POMS total scores and PSQI-K scores. Conclusion: Certain individuals with OSA are more likely to suffer from disturbed mood states without serious OSA indices. Psychiatric manifestations should also be considered when determining the severity of the disorder.
\end{abstract}

Keywords: Obstructive sleep apnea; Cluster analysis; Psychiatry; Fatigue

Received: August 19, 2021 Revised: September 7, 2021 Accepted: September 8, 2021

Corresponding author: Eui-Joong Kim, MD, PhD, Department of Psychiatry, Nowon Eulji Medical Center, Eulji University, 68 Hangeulbiseok-ro, Nowon-gu, Seoul 01830, Korea.

Tel: 82-2-970-8603, Fax: 82-2-949-2356, E-mail: kimej@eulji.ac.kr

(a) This is an Open Access article distributed under the terms of the Creative Commons Attribution Non-Commercial License (https://creativecommons.org/licenses/bync/4.0) which permits unrestricted non-commercial use, distribution, and reproduction in any medium, provided the original work is properly cited.

\section{INTRODUCTION}

Obstructive sleep apnea (OSA), characterized by frequent sleep fragmentations due to partial or complete obstruction of the upper airway resulting in intermittent hypoxia, is a complex disorder that affects not only one's quality of sleep but also leaves a lasting impact on one's quality of life. According to the American Academy of Sleep Medicine, OSA can be diagnosed when a patient presents an apnea-hypopnea index (AHI) $\geq 5$ with associated symptoms or an $\mathrm{AHI} \geq 15$ regardless of associated symptoms [1].

OSA is estimated to affect about $3 \%-7 \%$ of men, $2 \%-5 \%$ of women, and these numbers are expected to rise globally owing to an increase in obesity [2,3]. There is a growing health concern for OSA due to its correlation with cardiovascular and metabolic diseases such as hypertension, stroke, and diabetes [4-6]. Evidence suggests that it is also associated with an increased risk of mortality and morbidity due to such diseases $[7,8]$. Physiological change is not the only negative consequence of OSA that we should be aware of; negative effects on psychiatric health should also be dealt with. Symptoms of OSA include daytime sleepiness, fatigue, and unrefreshing sleep, and these symptoms can often impact one's quality of life [9]. Evidence from previous studies indicates that nonrestorative sleep and consequent daytime sleepiness are linked with depression or unstable mood [10-13]. When diagnosing OSA, the aforementioned consequences of the disease should also be taken into consideration since it can lead to 
such serious consequences on one's physical and mental health.

Unfortunately, due to the heterogeneity of the disorder, no clear subgroups or phenotypes have been established making it difficult to predict prognosis [14]. This is where cluster analysis, which recognizes similar characteristics and groups them, comes in handy [15]. The most notable cluster analysis study done on OSA was the Icelandic Sleep Apnea Cohort (ISAC) that identified three subgroups-disturbed sleep group, minimally symptomatic group, and excessive daytime sleepiness group-using validated sleep-related questionnaires in addition to comorbid conditions [15]. Several other studies from Europe and Asia ensued clustering based on multiple polysomnography (PSG) variables, demographic factors, sleep-related symptoms, comorbidities, and some studies obtained similar results whereas others ended up with different clusters [16-19].

Previous studies, however, have not explored psychiatric or mood symptoms related to OSA in-depth although sleep-related symptoms can markedly impact one's mood. To assess this aspect of OSA, we aimed not only to identify subgroups of OSA by applying cluster analysis but also attempted to show how mood problems manifest in subgroups of OSA.

\section{METHODS}

\section{Subjects}

A total of 1,446 participants underwent PSG at Nowon Eulji Medical Center (Seoul, Korea) from January 2003 to November 2012. This retrospective study included adult Korean patients who met the diagnostic criteria of OSA. They also had to fill out the following self-report questionnaires: Epworth Sleepiness Scale (ESS), Korean version of the Pittsburgh Sleep Quality Index (PSQI-K), Horne and Östberg Questionnaire (HOQ), and Korean edition of Profile of Mood States (K-POMS). Those with missing data were excluded from the study, and as a result, this study comprised 346 OSA patients. This study was approved by the Institutional Review Board of Eulji Hospital (Eulji 2017-06-011), and written consent was obtained from all participants.

\section{Polysomnography}

All subjects underwent overnight PSG (Medcare-Embla ${ }^{\circledR}$, A10, S7000; Reykjavík, Iceland) at Nowon Eulji Medical Center. Somnologica (Medcare-Embla ${ }^{\circledR}$ ) software program was used for analysis. A trained technician did a preliminary scoring of PSG variables, and then psychiatrists with specialties in sleep medicine confirmed scorings according to the American Academy of Sleep Medicine Scoring Manual. Apneas were defined as an absence of airflow on the oronasal thermistor or nasal pressure cannula for $\geq 10 \mathrm{sec}$. Hypopneas were defined as a $\geq 30 \%$ reduction from baseline in airflow for $\geq 10 \mathrm{sec}$ associated with at least 3\% oxygen desaturation. The AHI was calculated as the mean number of apneas and hypopneas per hour of sleep [1]. The following were also obtained in PSG: respiratory disturbance index (RDI), oxygen desaturation index (ODI), sleep efficiency, respiratory effort-relat- ed arousal (RERA), mean oxygen saturation, lowest oxygen saturation, time percentage of $<90 \%$ saturation, average desaturation percentage. According to the third edition of the International Classification of Sleep Disorders, RDI is defined as the sum of apneas, hypopneas, RERAs divided by total sleep time [20]. RDI $\geq 5$ per hour of sleep coupled with associated symptoms, medical, or psychiatric disorders are required for a diagnosis of OSA. Individuals with an $\mathrm{RDI} \geq 15$ regardless of associated symptoms can be also diagnosed as having OSA [20].

\section{Symptom questionnaire}

To better understand subjective sleep quality and sleep-related symptoms, it was important to utilize valid self-report instruments. ESS, PSQI-K, HOQ, and K-POMS were used as self-report questionnaires.

The ESS is a widely known scale that assesses daytime sleepiness. It measures eight items that can range from 0 to 3 , making the total score from 0 to 24 . The higher the score, the more likely one suffers from daytime sleepiness [21]. The interpretations of the ESS scores are as follows: 0-10, normal daytime sleepiness; 11-24, excessive daytime sleepiness [21]. We used the Korean version of ESS which was validated for reliability by Cho et al. [22].

The PSQI-K is comprised of nine questions that evaluate overall sleep habits in the past month [23]. Specific components are as follows: sleep quality, sleep latency, sleep duration, habitual sleep efficiency, sleep disturbances, use of sleep medication, and daytime dysfunction. Each component can range from 0 to 3 , and the total score ranges from 0 to 21 . The higher the score, the worse one's sleep quality is. The PSQI cutoff point for poor sleepers is 5 or above [24], but according to Sohn et al. [23], 8.5 has been suggested for the best cutoff point for PSQI-K.

The HOQ analyzes one's morningness or eveningness with 19 questions with scores ranging from 16 to 86 . Scores of 41 and below indicate "evening types", scores of 59 and above indicate "morning types," scores between 42 and 58 indicate "intermediate types" [25].

The K-POMS, unlike other depression scales, is a test that measures the entirety of mood by measuring six traits: tension, depression, anger, vigor, fatigue, and confusion. The K-POMS total score, also known as total mood disturbances, is calculated according to the following equation: (Tension+Depression+Anger+ Fatigue+Confusion) - Vigor [26].

\section{Statistical analysis}

The cluster analysis was performed using K-means cluster analysis. For numerical variables, the results were expressed as the mean value \pm standard deviation, and for nominal variables, the results were expressed as percentages. Intergroup differences between clusters were evaluated using one-way ANOVA and post hoc Bonferroni analysis. A value of $\mathrm{p}<0.05$ was defined as statistically significant. All statistical analyses were performed using SPSS ver. 22 (IBM Corp., Armonk, NY, USA). 


\section{RESULTS}

\section{Demographics}

A total of 346 patients were analyzed, and this study mainly consisted of middle-aged men with severe OSA. The mean age was $47.07 \pm 13.00$ years, and the mean AHI was $37.78 \pm 24.05$. The

Table 1. Descriptive characteristics of the study sample

\begin{tabular}{|c|c|}
\hline Variables & Values $(\mathrm{n}=346)$ \\
\hline Age (yr) & $47.07 \pm 13.00$ \\
\hline Male gender & $267(77.17)$ \\
\hline $\mathrm{AHI}(/ \mathrm{hr})$ & $37.78 \pm 24.05$ \\
\hline $\mathrm{BMI}\left(\mathrm{m}^{2} / \mathrm{kg}\right)$ & $25.96 \pm 3.87$ \\
\hline ESS & $8.94 \pm 5.04$ \\
\hline HOQ & $52.23 \pm 9.73$ \\
\hline K-POMS total score & $25.60 \pm 35.52$ \\
\hline
\end{tabular}

Data are presented as mean \pm SD or $\mathrm{n}(\%)$. AHI, apnea-hypopnea index; BMI, body mass index; ESS, Epworth Sleepiness Scale; HOQ, Horne and Östberg Questionnaire; K-POMS, Korean edition of Profile of Mood States average BMI was $25.96 \pm 3.87$, and the average ESS score was $8.94 \pm$ 5.04. They were mostly intermediate types in terms of morningness or eveningness with the HOQ of 52.23 \pm 9.73 . The average K-POMS total score was $25.60 \pm 35.52$. The general characteristics of this study's population are displayed in Table 1.

\section{Cluster analyses}

A total of 11 PSG variables were taken into consideration along with BMI, age, and results of self-report questionnaires mentioned above. Cluster analyses resulted in three distinct subgroups in our study population as presented in Table 2. Age, hypopnea index, and sleep efficiency were not statistically significant. The scores of the HOQ indicate that all patients were intermediate types.

Cluster 1, with 166 patients, turned out to be the largest cluster among the three clusters (47.98\%) and similar to other clusters, was predominantly male $(\mathrm{n}=124)$. The mean ESS score was $8.06 \pm$ 4.90 , and the mean PSQI-K score was 7.27 \pm 4.15 . This group had the lowest K-POMS total score $(7.99 \pm 18.59)$ and the lowest AHI (24.81 \pm 13.06$)$. PSG indices such as RDI, ODI, saturation values

Table 2. Clinical characteristics of three subtypes after cluster analysis

\begin{tabular}{|c|c|c|c|c|c|}
\hline Characteristics & Cluster 1 & Cluster 2 & Cluster 3 & p-value & $\begin{array}{l}\text { Bonferroni } \\
\text { correction }\end{array}$ \\
\hline Number of patients & $166(47.98)$ & $90(26.01)$ & $90(26.01)$ & & \\
\hline Male & $124(74.70)$ & $81(90.00)$ & $62(68.89)$ & & - \\
\hline Age & $48.27 \pm 13.72$ & $44.58 \pm 9.83$ & $47.37 \pm 14.17$ & 0.093 & \\
\hline ESS & $8.06 \pm 4.90$ & $10.14 \pm 4.44$ & $9.34 \pm 5.59$ & 0.004 & $1,3<2$ \\
\hline PSQI-K & $7.27 \pm 4.15$ & $6.97 \pm 3.39$ & $10.46 \pm 4.33$ & $<0.001$ & $1,2<3$ \\
\hline HOQ & $53.61 \pm 10.60$ & $50.92 \pm 8.69$ & $50.99 \pm 8.73$ & 0.040 & - \\
\hline K-POMS total score & $7.99 \pm 18.59$ & $9.39 \pm 21.05$ & $74.27 \pm 23.84$ & $<0.001$ & $1,2<3$ \\
\hline POMS-T & $2.40 \pm 4.98$ & $2.10 \pm 4.44$ & $13.10 \pm 6.51$ & $<0.001$ & $1,2<3$ \\
\hline POMS-D & $5.80 \pm 6.56$ & $5.48 \pm 5.93$ & $23.63 \pm 10.86$ & $<0.001$ & $1,2<3$ \\
\hline POMS-A & $4.45 \pm 5.31$ & $4.99 \pm 5.03$ & $16.89 \pm 7.71$ & $<0.001$ & $1,2<3$ \\
\hline POMS-V & $12.63 \pm 6.88$ & $12.46 \pm 6.69$ & $8.18 \pm 4.92$ & $<0.001$ & $1,2<3$ \\
\hline POMS-F & $7.36 \pm 4.58$ & $7.20 \pm 4.59$ & $15.93 \pm 5.03$ & $<0.001$ & $1,2<3$ \\
\hline POMS-C & $2.87 \pm 3.58$ & $2.61 \pm 3.16$ & $11.54 \pm 4.07$ & $<0.001$ & $1,2<3$ \\
\hline $\mathrm{BMI}\left(\mathrm{m}^{2} / \mathrm{kg}\right)$ & $25.14 \pm 3.16$ & $27.85 \pm 4.21$ & $25.59 \pm 4.11$ & $<0.001$ & $1,3<2$ \\
\hline $\mathrm{AHI}(/ \mathrm{hr})$ & $24.81 \pm 13.06$ & $68.35 \pm 16.13$ & $31.14 \pm 19.02$ & $<0.001$ & $1,3<2$ \\
\hline $\mathrm{RDI}(/ \mathrm{hr})$ & $25.52 \pm 13.10$ & $68.51 \pm 16.05$ & $32.01 \pm 18.60$ & $<0.001$ & $1<3<2$ \\
\hline Hypopnea index (/hr) & $14.43 \pm 9.88$ & $17.96 \pm 16.37$ & $16.65 \pm 11.73$ & 0.076 & - \\
\hline Apnea index $(/ \mathrm{hr})$ & $10.33 \pm 8.98$ & $50.27 \pm 21.04$ & $14.48 \pm 14.89$ & $<0.001$ & $1,3<2$ \\
\hline ODI (/hr) & $15.64 \pm 10.16$ & $56.98 \pm 17.23$ & $22.38 \pm 15.82$ & $<0.001$ & $1<3<2$ \\
\hline Sleep efficiency (\%) & $82.57 \pm 13.17$ & $85.02 \pm 8.65$ & $82.09 \pm 15.03$ & 0.234 & - \\
\hline RERA (/hr) & $0.98 \pm 1.33$ & $0.16 \pm 0.45$ & $0.86 \pm 2.09$ & $<0.001$ & $1,3<2$ \\
\hline Mean oxygen saturation (\%) & $95.64 \pm 1.26$ & $92.66 \pm 2.93$ & $94.87 \pm 2.76$ & $<0.001$ & $1<3<2$ \\
\hline Lowest oxygen saturation (\%) & $81.78 \pm 8.64$ & $72.44 \pm 9.34$ & $80.43 \pm 7.63$ & $<0.001$ & $1,3<2$ \\
\hline $\begin{array}{l}\text { Time percentage of } \\
<90 \% \text { saturation }(\%)\end{array}$ & $2.04 \pm 3.67$ & $20.91 \pm 17.93$ & $3.87 \pm 5.32$ & $<0.001$ & $1,3<2$ \\
\hline $\begin{array}{l}\text { Average desaturation } \\
\text { percentage }(\%)\end{array}$ & $6.12 \pm 1.49$ & $10.13 \pm 3.40$ & $6.45 \pm 1.64$ & $<0.001$ & $1,3<2$ \\
\hline
\end{tabular}

Data are presented as n (\%) or mean \pm SD. ESS, Epworth Sleepiness Scale; PSQI-K, Korean version of the Pittsburgh Sleep Quality Index; HOQ, Horne and Östberg Questionnaire; K-POMS, Korean edition of Profile of Mood States; POMS-T, Profile of Mood States-Tension; POMS-D, Profile of Mood States-Depression; POMS-A, Profile of Mood States-Anger, POMS-V, Profile of Mood States-Vigor; POMS-F, Profile of Mood StatesFatigue; POMS-C, Profile of Mood States-Confusion; BMI, body mass index; AHI, apnea-hypopnea index; RDI, respiratory disturbance index; ODI, oxygen desaturation index; RERA, respiratory effort-related arousal 
were the least severe when compared to other clusters. Thus, this cluster was labeled as the "moderately symptomatic OSA with the least disturbed mood group."

Cluster 2 ( $\mathrm{n}=90,26.01 \%)$ corresponded to severe symptoms of OSA (AHI 68.35 \pm 16.13 ) with excessive daytime sleepiness (ESS, 10.14 \pm 4.44$)$. Several PSG indices showed that patients who belonged to this group had the worst breathing and saturation parameters. The mean RDI was $68.51 \pm 16.05$. The average desaturation percentage was $10.13 \pm 3$.40. It should be noted that cluster 2 had the highest BMI (27.85 \pm 4.21$)$ perhaps accounting for the severity of OSA parameters. Although patients in this cluster complained of daytime sleepiness, their K-POMS total scores do not indicate disturbed mood (9.39 \pm 21.05$)$. This group was labeled as the "severely symptomatic OSA not so disturbed mood group."

Cluster 3 was the same size as cluster $2(n=90,26.01 \%)$ but had more female population (cluster $2, \mathrm{n}=9$ vs. cluster $3, \mathrm{n}=18$ ). Although the ESS scores $(9.34 \pm 5.59)$ were in between those of clusters 1 and 2, patients in this cluster had the highest K-POMS total scores (74.27 \pm 23.84 ) and complained of poor sleep quality (PSQI-K $10.46 \pm 4.33)$. When analyzing subscales of the K-POMS total scores, cluster 3 patients showed mood disturbances in all six traits compared to patients who belonged to other groups. The mean AHI was $31.14 \pm 19.02$. The mean ODI was $22.38 \pm 15.82$, and the mean $\mathrm{RDI}$ was $32.01 \pm 18.60$. When compared to cluster 2 , all PSG variables indices were better in quality for cluster 3 . The final cluster was labeled as the "moderately symptomatic with highest disturbed mood group."

\section{DISCUSSION}

Currently, when classifying OSA, only the AHI index is taken into consideration, and there has been a large amount of evidence that supports the index alone is not enough to understand the complex and heterogeneous nature of the disorder. The ISAC study was the first to perform cluster analysis on its study population in an attempt to identify subgroups of OSA. Several other studies tested the generalizability and replicability of the findings in the ISAC study to establish universally applicable subgroups. Although some subgroups looked promising, it is still an ongoing process. This study was also developed to integrate various aspects of the disorder, particularly focusing on the psychiatric outcomes of OSA. After incorporating OSA severity, symptoms related to sleep, mood states, and several PSG variables, this study identified three clusters for 346 OSA patients.

Cluster 1, the moderately symptomatic OSA with the least disturbed mood group, in general had the lowest average ESS, PSQI$\mathrm{K}$, K-POMS total scores, AHI, RDI, ODI, RERA, and saturation indices compared to clusters 2 and 3. These numbers are especially substantial when compared to cluster 2 , the severely symptomatic OSA not so disturbed mood group. As represented in Figure 1 , clusters 1 and 2 are distinctive from each other when considering K-POMS total scores and AHI. The different characteristics of clusters 1 and 2 are also evident in post hoc analysis; cluster 2 was

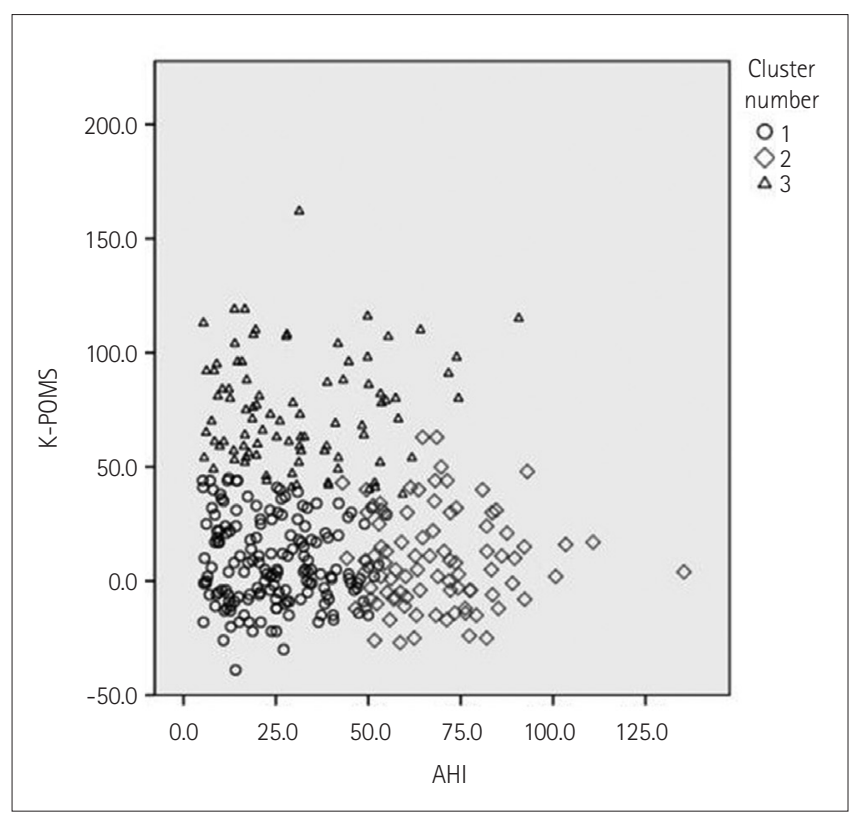

Figure 1. Representation of three clusters after k-means cluster analysis.

significantly different from cluster 1 in ESS, BMI, AHI, RDI, ODI, RERA, and all saturation indices. These parameters are related to the severity of OSA symptoms and thus explains excessive daytime sleepiness patients in cluster 2 complain. However, despite these differences, K-POMS total scores between clusters 1 and 2 are not significantly different from each other after post hoc analysis. A similar result was obtained when comparing PSQI-K scores.

Cluster 3, the moderately symptomatic with highest disturbed mood group, compared to cluster 1 had higher average ESS, PSQI$\mathrm{K}$, K-POMS total scores, BMI, AHI, RDI, ODI, RERA, mean oxygen saturation, lowest oxygen saturation, time percentage of $<90 \%$ saturation, average desaturation percentage. However, after post hoc analysis, there was no significant difference between most values except for PSQI-K, K-POMS total scores, RDI, ODI, mean oxygen saturation. Although clusters 1 and 3 display similar profiles, patients in cluster 3 experienced poorer sleep quality and presented more mood disturbances.

When comparing cluster 2 with 3, despite having less severe AHI and saturation indices, patients in cluster 3 scored worse PSQI-K and K-POMS total scores compared to patients in cluster 2 . The lack of correlation between one's subjective feeling of poor sleep quality and objective values such as PSG values implies that patients in cluster 3 are experiencing subjective-objective sleep discrepancy, a common finding in insomnia disorders [27]. There was no significant difference between each cluster in terms of sleep efficiency: cluster 1, mean $82.57 \% \pm 13.17 \%$; cluster 2 , mean $85.02 \% \pm$ $8.65 \%$; and cluster 3 , mean $82.09 \% \pm 15.03 \%$. However, individuals in cluster 3 seem more vulnerable to poor sleep quality and display the highest K-POMS total scores, POMS-T (tension), POMS-D (depression), POMS-A (anger), POMS-F (fatigue), and POMS-C (confusion) scores, along with the lowest POMS-V (vigor). Whether poor sleep quality contributes to poor mood states, or it is the 
poor mood states that contributes to poor sleep quality could not be distinguished in this study and leaves room for future studies.

Our study has demonstrated the even with similar or better PSG profiles do not always correlate with lesser fatigue or better mood in the population OSA. Considering the heterogeneity of the disorder this result does not come as a surprise and once again recognizes the need to better understand the disorder. In the psychiatric context, sleep not only has been associated with the ability to carry out daytime activities but also with depression and numerous psychiatric disorders. One can experience fatigue as a symptom of depression or as a result of being tired, and it is often hard to differentiate the cause. In our study, fatigue and depression were separately measured, and it showed that individuals who had the highest mood disturbances measured by K-POMS also exhibited the highest POMS-D and POMS-F. The study also showed that these individuals' ESS scores, which measured daytime sleepiness, were not associated with K-POMS total scores.

\section{Strengths and limitations}

To the best of our knowledge, this was the first study to include mood states in cluster analysis of OSA. Our study not only attempted to cluster OSA according to previously utilized parameters but also focused on psychiatric outcomes related to OSA. Our study showed that there is a certain population within OSA patients who are more affected psychiatrically. They exhibited significantly higher mood disturbances as demonstrated in KPOMS total scores and its six components. Previous studies have shown a higher prevalence of depression in OSA patients compared with the general population [28]. Other studies have suggested depression as a risk factor for coronary artery disease [29]. Thus, higher mood disturbance combined with OSA is likely to contribute to an increase in morbidity and mortality of OSA. Our study's results suggest when evaluating the severity of OSA, psychiatric presentations, such as mood disturbance, should also be taken into consideration, along with the conventional parameters such as AHI and ODI. We also suggest that in future studies, the validity and efficacy of treatment for disturbed mood along with treatment for OSA to be explored in a psychiatrically vulnerable population of OSA patients.

The study has several limitations. First, this study was a singlecenter study that comprised of Korean patients and therefore not ethnically diverse. Some characteristics of OSA, such as craniofacial profile or BMI, are different between the Asian population and the non-Asian population, and the generalizability of this study might not extend to the non-Asian population [30]. Second, the accessibility of PSG may have caused selection bias. Before 2018, PSG was not covered by the national health insurance system in Korea, and there is a chance that individuals with mild OSA symptoms were less likely to get tested than those with severe OSA symptoms. Lastly, PSG only gathers data from a single night whereas the ESS, PSQI-K, HOQ, and K-POMS total scores are representations of a longer period. This leaves room for discrepancies between PSG indices and the self-report scales, and thus, future studies should consider follow-up PSG.

\section{Conclusion}

Our study showed three distinct clusters: the moderately symptomatic OSA with the least disturbed mood group, the severely symptomatic OSA not so disturbed mood group, and the moderately symptomatic OSA with highest disturbed mood group. Comparison between clusters indicated that there are individuals who are more likely to complain about disturbed mood states among OSA patients. Since OSA can have a serious impact on one's quality of life, mood states and its psychiatric outcomes should also be important factors when diagnosing the disorder.

\section{Acknowledgments}

None

\section{Conflicts of Interest}

The authors have no potential conflicts of interest to disclose.

\section{Author Contributions}

Conceptualization: Hyo Jin Kim, Soo-Young Bhang, Jae-Won Choi. Data curation: Eui-Joong Kim, Jae-Won Choi. Formal analysis: Hyo Jin Kim. Methodology: Hyo Jin Kim, Kyu Young Lee, EunJeong Joo, Eui-Joong Kim. Writing_original draft: Hyo Jin Kim, Eui-Joong Kim. Writing—review \& editing: Hyo Jin Kim, EuiJoong Kim, Jae-Won Choi.

\section{ORCID iDs}

Hyo Jin Kim (1)

https://orcid.org/0000-0003-2906-3016

Jae-Won Choi (1)

https://orcid.org/0000-0001-6294-7046

Eun-Jeong Joo (i)

https://orcid.org/0000-0001-8766-8713

Kyu Young Lee (1)

https://orcid.org/0000-0003-3214-6082

Soo-Young Bhang 10

https://orcid.org/0000-0001-5254-0314

Eui-Joong Kim (1)

https://orcid.org/0000-0002-0156-1964

\section{REFERENCES}

1. Berry RB, Brooks R, Gamaldo C, Harding SM, Lloyd RM, Quan SF, et al. AASM scoring manual updates for 2017 (version 2.4). J Clin Sleep Med 2017;13:665-666.

2. Schwartz AR, Patil SP, Laffan AM, Polotsky V, Schneider H, Smith PL. Obesity and obstructive sleep apnea: pathogenic mechanisms and therapeutic approaches. Proc Am Thorac Soc 2008;5:185-192.

3. Jehan S, Zizi F, Pandi-Perumal SR, Wall S, Auguste E, Myers AK, et al. Obstructive sleep apnea and obesity: implications for public health. Sleep Med Disord 2017;1:00019.

4. Labarca G, Cruz NR, Descalzi F. Multisystemic involvement in obstructive sleep apnea. Rev Med Chil 2014;142:748-757.

5. Bradley TD, Floras JS. Obstructive sleep apnoea and its cardiovascular consequences. Lancet 2009;373:82-93.

6. Shahar E, Whitney CW, Redline S, Lee ET, Newman AB, Nieto FJ, et al. 
Sleep-disordered breathing and cardiovascular disease: cross-sectional results of the sleep heart health study. Am J Respir Crit Care Med 2001;163: 19-25.

7. Robichaud-Hallé L, Beaudry M, Fortin M. Obstructive sleep apnea and multimorbidity. BMC Pulm Med 2012;12:60.

8. Marshall NS, Wong KK, Cullen SR, Knuiman MW, Grunstein RR. Sleep apnea and 20-year follow-up for all-cause mortality, stroke, and cancer incidence and mortality in the Busselton Health Study Cohort. J Clin Sleep Med 2014;10:355-362.

9. Baldwin CM, Griffith KA, Nieto FJ, O'Connor GT, Walsleben JA, Redline S. The association of sleep-disordered breathing and sleep symptoms with quality of life in the sleep heart health study. Sleep 2001;24:96-105.

10. Ejaz SM, Khawaja IS, Bhatia S, Hurwitz TD. Obstructive sleep apnea and depression: a review. Innov Clin Neurosci 2011;8:17-25.

11. Andrews JG, Oei TP. The roles of depression and anxiety in the understanding and treatment of obstructive sleep apnea syndrome. Clin Psychol Rev 2004;24:1031-1049.

12. Sharafkhaneh A, Giray N, Richardson P, Young T, Hirshkowitz M. Association of psychiatric disorders and sleep apnea in a large cohort. Sleep 2005; 28:1405-1411.

13. Baran AS, Richert AC. Obstructive sleep apnea and depression. CNS Spectr 2003;8:128-134.

14. Kim SJ, Alnakhli WM, Alfaraj AS, Kim KA, Kim SW, Liu SY. Multi-perspective clustering of obstructive sleep apnea towards precision therapeutic decision including craniofacial intervention. Sleep Breath 2021;25:85-94.

15. Ye L, Pien GW, Ratcliffe SJ, Björnsdottir E, Arnardottir ES, Pack AI, et al. The different clinical faces of obstructive sleep apnoea: a cluster analysis. Eur Respir J 2014;44:1600-1607.

16. Vavougios GD, Ntaios G, Pastaka C, Zarogiannis SG, Gourgoulianis KI. Phenotypes of comorbidity in OSAS patients: combining categorical principal component analysis with cluster analysis. J Sleep Res 2016;25:31-38.

17. Lacedonia D, Carpagnano GE, Sabato R, Lo Storto MM, Patricelli G, Foschino Barbaro MP, et al. Application of cluster analysis in OSA population. Eur Respir J 2016;48(Suppl 60):PA2311.

18. Kim J, Keenan BT, Lim DC, Lee SK, Pack AI, Shin C. Symptom-based subgroups of Koreans with obstructive sleep apnea. J Clin Sleep Med 2018;14:
437-443.

19. Kim JW, Won TB, Rhee CS, Park YM, Yoon IY, Cho SW. Polysomnographic phenotyping of obstructive sleep apnea and its implications in mortality in Korea. Sci Rep 2020;10:13207.

20. American Academy of Sleep Medicine. International classification of sleep disorders. 3rd. Darien, IL: American Academy of Sleep Medicine; 2014.

21. Smith SS, Oei TP, Douglas JA, Brown I, Jorgensen G, Andrews J. Confirmatory factor analysis of the Epworth Sleepiness Scale (ESS) in patients with obstructive sleep apnoea. Sleep Med 2008;9:739-744.

22. Cho YW, Lee JH, Son HK, Lee SH, Shin C, Johns MW. The reliability and validity of the Korean version of the Epworth sleepiness scale. Sleep Breath 2011;15:377-384.

23. Sohn SI, Kim DH, Lee MY, Cho YW. The reliability and validity of the Korean version of the Pittsburgh Sleep Quality Index. Sleep Breath 2012;16:803812 .

24. Buysse DJ, Reynolds CF 3rd, Monk TH, Berman SR, Kupfer DJ. The Pittsburgh Sleep Quality Index: a new instrument for psychiatric practice and research. Psychiatry Res 1989;28:193-213.

25. Horne JA, Ostberg O. A self-assessment questionnaire to determine morningness-eveningness in human circadian rhythms. Int J Chronobiol 1976;4: 97-110.

26. Kim EJ, Lee SI, Jeong DU, Shin MS, Yoon IY. Standardization and reliability and validity of the Korean edition of Profile of Mood States (K-POMS). Sleep Med Psychophysiol 2003;10:39-51.

27. Rezaie L, Fobian AD, McCall WV, Khazaie H. Paradoxical insomnia and subjective-objective sleep discrepancy: a review. Sleep Med Rev 2018;40: 196-202.

28. Jehan S, Auguste E, Pandi-Perumal SR, Kalinowski J, Myers AK, Zizi F, et al. Depression, obstructive sleep apnea and psychosocial health. Sleep Med Disord 2017;1:00012.

29. Khawaja IS, Westermeyer JJ, Gajwani P, Feinstein RE. Depression and coronary artery disease: the association, mechanisms, and therapeutic implications. Psychiatry (Edgmont) 2009;6:38-51.

30. Lee RW, Vasudavan S, Hui DS, Prvan T, Petocz P, Darendeliler MA, et al. Differences in craniofacial structures and obesity in Caucasian and Chinese patients with obstructive sleep apnea. Sleep 2010;33:1075-1080. 